\title{
Clinical study in patients with ocular ischemic diseases treated with enhanced external counterpulsation combined with drugs
}

\author{
YUXIN YANG $^{1}$, HAITAO ZHANG $^{1}$, YITAO YAN ${ }^{1}$ and YANLING GUI ${ }^{2}$ \\ Departments of ${ }^{1}$ Ophthalmology and ${ }^{2}$ Nursing, The First Affiliated Hospital of \\ Xinxiang Medical University, Weihui, Henan 453100, P.R. China
}

Received November 15, 2012; Accepted April 20, 2013

DOI: $10.3892 / \mathrm{mmr} .2013 .1445$

\begin{abstract}
The aim of this study was to evaluate the clinical effect in patients with ocular ischemic diseases treated with enhanced external counterpulsation (EECP) combined with drugs. A total of 65 patients with carotid artery stenosis were included in this study. Group A consisted of 31 patients (43 eyes) treated with EECP and medication, and group B consisted of 34 patients (49 eyes) treated with medication alone. The clinical effect was analyzed by comparing the visual acuity, visual fields and optical hemodynamics between the two groups of patients. Ocular ischemic diseases mainly included ischemic optic neuropathy, central (branch) retinal artery occlusion, ophthalmoplegia externa and ocular ischemic syndrome. Significant improvement of visual acuity, visual fields and optical hemodynamics was observed in the patients of group A, and statistically significant differences were found between groups $\mathrm{A}$ and $\mathrm{B}\left(\chi^{2}=4.935,7.124\right.$ and 5.478, respectively; $\mathrm{P}<0.05)$. In conclusion, ophthalmologists should observe for ocular ischemic diseases. The symptoms of the disease and the vision of the patient could be effectively improved by EECP, which has no evident complications.
\end{abstract}

\section{Introduction}

Enhanced external counterpulsation (EECP) was developed in 1980 by Zheng in The First Affiliated Hospital of Sun Yat-Sen University (Guangzhou, China). Following this, EECP has been used to treat ischemic diseases such as coronary heart disease and angina pectoris, following percutaneous coronary intervention, transplantation and coronary artery bypass grafting, cerebral arteriosclerosis, cerebral ischemia, embolism of retinal artery, ischemic optic neuropathy, optic atrophy and hearing loss (1-4).

Correspondence to: Dr Yuxin Yang, Department of Ophthalmology, The First Affiliated Hospital of Xinxiang Medical University, No. 88 Jiankang Road, Weihui, Henan 453100, P.R. China E-mail: yuxinyangcn@126.com

Key words: enhanced external counterpulsation, ocular ischemic disease, curative effect
Patients with ocular ischemic diseases have been previously treated with EECP and medication in the Department of Ophthalmology, the First Affiliated Hospital of Sun Yat-Sen University since 1985 . In the present study, we retrospectively analyzed the clinical data of 65 patients (92 eyes) with carotid artery stenosis treated with EECP and medication.

\section{Subjects and methods}

Subjects. A total of 65 patients with carotid artery stenosis examined between January, 2005 and May, 2009 were included in the present study. This study was conducted in accordance with the Declaration of Helsinki and was approved by the Ethics Committee of the First Affiliated Hospital of Xinxiang Medical University (Weihui, China). Written informed consent was obtained from all the participants. There were 31 male and 34 female patients with a mean age of $64.17 \pm 8.25$ years (range, 29-86 years). Diagnoses were confirmed by examination of visual acuity, slit lamp, ophthalmoscope, field of vision, fluorescein fundus angiography (FFA) test, intraocular pressure, visual evoked potential (VEP), optical ultrasound, X-ray and carotid artery color Doppler ultrasound. Patients diagnosed with ocular ischemic diseases were included in this study, while patients with additional primary ophthalmological conditions such as primary glaucoma, iridocyclitis, retinal vein occlusion, high myopia, retinitis pigmentosa, choroiditis, diabetic retinopathy, and other congenital fundus abnormalities were excluded.

Methods. The clinical data of all the patients $(n=65)$ were retrospectively analyzed. Group A consisted of 31 patients (43 eyes) treated with EECP and medication, and group B consisted of 34 patients (49 eyes) treated with medication alone. The clinical effect in the patients of the two groups was evaluated by comparing the visual acuity, visual fields and optical hemodynamics.

Patient assessment. Patient assessment included examination of visual acuity, field of vision (Zeiss 750 Visual Field Analyzer) and optical hemodynamics prior to and following treatment. Color Doppler ultrasound (Philips HD7) was used to examine ophthalmic arterial hemodynamics with a frequency of $10 \mathrm{MHz}$. Hemodynamic parameters in the ophthalmic artery of each patient were recorded, including 
peak systolic blood flow velocity (Psv), end-diastolic blood flow velocity $(\mathrm{Edv})$, mean blood flow velocity $(\mathrm{Vm})$, resistance index (RI) and pulse index (PI). Each parameter was measured three times and the mean value was used.

Treatment. The 31 patients (43 eyes) in group A were treated with EECP and medication for 2 months. In group B, 34 patients (49 eyes) were treated with medication alone for 2 months for regulation of blood pressure, regulation of blood sugar, neurotrophy, anticoagulation and improvement of microcirculation.

Thirty-one patients in group A were treated with EECP once a day for $1 \mathrm{~h}$, and the course of treatment lasted for a half to 2 months. Balloon pumping pressure $\left(0.35-0.4 \mathrm{MPa} / \mathrm{cm}^{2}\right)$ utilized in younger patients whose course of disease was $<3$ months were treated with EECP twice a day. Patients who had who had undergone bypass surgery or had suffered heart attack, severe heart failure, congestive heart disease, aortic disease, aortic incompetence, abdominal aortic aneurysm (aaa), severe peripheral vascular disease, frequent arrhythmia and venous thrombosis, were excluded from group A.

Curative effect standards. Curative effect standards were applied when one of the following conditions occurred: i) The patient could read a minimum of three lines on a visual acuity chart, with visual fields $>10 \mathrm{~b}$, and significant improvement with hemodynamic parameters $(\mathrm{P}<0.01)$; ii) patients could read a minimum of two lines on the visual acuity chart, with visual fields $>5$ b or improved hemodynamic parameters $(\mathrm{P}<0.05)$; iii) patients did not exhibit improved visual acuity, visual fields and optical hemodynamics (5-7).

Statistical analysis. Statistical analysis was performed using SPSS v.14 (SPSS, Inc., Chicago, IL, USA). An independent sample t-test and $\chi^{2}$ test were used to compare the differences between the groups. Univariate and unconditional logistic stepwise regression methods were used to adjust for confounding factors and analyze the interactions between factors. Factors affecting outcome regarding the disease were not considered.

\section{Results}

Types of ocular ischemic diseases of the included patients. The types of ocular ischemic diseases of the patients included in this study were ischemic optic nerve disorders, ocular ischemic syndrome, retinal central (branch) vein occlusion and external ophthalmoplegia (Table I).

Statistically significant differences between groups $A$ and $B$. All the patients with ocular ischemic diseases were treated with EECP and medication for two mouths. The total effective rate in group A was $86.05 \%$ (37 eyes), while the rate in group B was $63.27 \%$ (31 eyes), indicating statistically significant differences between groups $\mathrm{A}$ and $\mathrm{B}\left(\chi^{2}=2.238, \mathrm{P}<0.05\right)$ (Tables II-IV).

The present study identified a close positive correlation between the curative effect and the course of disease in the patients of group $\mathrm{A}(\mathrm{R}=0.719, \mathrm{P}<0.05)$. The correlation analysis revealed that the patients with a shorter course of disease, exhibited an improved curative effect (Table V).
Table I. Types of ocular ischemic diseases of the included patients $(n=65)$.

\begin{tabular}{lcc}
\hline Types of ocular ischemic diseases & $\begin{array}{c}\text { No. of } \\
\text { patients }\end{array}$ & $\begin{array}{c}\text { Constituent } \\
\text { ratio (\%) }\end{array}$ \\
\hline Ischemic optic nerve disorders & 24 & 36.92 \\
Ocular ischemic syndrome & 21 & 32.31 \\
Retinal central (branch) vein occlusion & 12 & 18.46 \\
External ophthalmoplegia & 8 & 12.31 \\
Total & 65 & \\
\hline
\end{tabular}

Table II. Comparison of visual acuity prior to and following treatment in the patients of group A.

\begin{tabular}{lccccc}
\hline & \multicolumn{5}{c}{ Visual acuity (m) } \\
\cline { 2 - 6 } Time & LP & $<0.1$ & $0.1-0.3$ & $0.4-0.9$ & $\geq 1.0$ \\
\hline Prior to treatment, $\mathrm{n}$ & 4 & 9 & 15 & 12 & 3 \\
Following treatment, $\mathrm{n}$ & 2 & 5 & 13 & 17 & 6 \\
\hline
\end{tabular}

LP, light perception.

Table III. Comparison of visual acuity prior to and following treatment in the patients of group B.

\begin{tabular}{lrrccc}
\hline & \multicolumn{5}{c}{ Visual acuity (m) } \\
\cline { 2 - 6 } Time & LP & $<0.1$ & $0.1-0.3$ & $0.4-0.9$ & $\geq 1.0$ \\
\hline Prior to treatment, $\mathrm{n}$ & 4 & 12 & 16 & 14 & 4 \\
Following treatment, $\mathrm{n}$ & 3 & 7 & 17 & 14 & 8 \\
\hline
\end{tabular}

LP, light perception.

Table IV. Comparison of curative effect between groups A and $\mathrm{B}$ (mean \pm standard deviation).

\begin{tabular}{lcccc}
\hline Groups & $\begin{array}{c}\text { No. of } \\
\text { patients }\end{array}$ & $\begin{array}{c}\text { No. of } \\
\text { eyes }\end{array}$ & $\begin{array}{c}\text { Effective } \\
\text { no. of eyes }\end{array}$ & $\begin{array}{r}\text { Effective } \\
\text { power (\%) }\end{array}$ \\
\hline A & 31 & 43 & 37 & 86.05 \\
B & 34 & 49 & 31 & 63.27 \\
\hline
\end{tabular}

$\chi^{2}=2.238, \mathrm{P}<0.05$.

Significantly reduced hemodynamic parameters in the patients of group A. To investigate the curative effect mechanism of EECP, we compared the hemodynamic parameters of all the patients prior to and following treatment. The results showed that ophthalmic artery perfusion of the patients in groups A and B was improved. Parameters including Psv, Edv and RI of the patients in group A were also reduced compared to those 
Table V. Association between curative effect and course of disease in the patients of group A.

\begin{tabular}{lcccc}
\hline Course of disease & No. of eyes & Excellence, $n$ & Effectiveness, $\mathrm{n}$ & Inefficiency, $\mathrm{n}$ \\
\hline 4 days to 1 month & 8 & 6 & 2 & 6 \\
2-3 months & 16 & 8 & 2 & 1 \\
$4-5$ months & 5 & 2 & 4 & 1 \\
6 months & 6 & 2 & 2 & 1 \\
1-2 years & 4 & 0 & 2 & 1 \\
5-10 years & 4 & 19 & 18 & 5 \\
Total & 43 & 1 & \\
\hline
\end{tabular}

Table VI. Comparison of hemodynamic parameters between groups A and B (mean \pm standard deviation).

\begin{tabular}{|c|c|c|c|c|c|c|c|c|}
\hline \multirow[b]{2}{*}{ Index } & \multicolumn{4}{|c|}{ Group A $(n=43)$} & \multicolumn{4}{|c|}{ Group B $(n=49)$} \\
\hline & Prior to treatment & Following treatment & t-value & $\mathrm{P}$-value & Prior to treatment & Following treatment & t-value & $\mathrm{P}$-value \\
\hline Psv & $9.14 \pm 2.25$ & $29.32 \pm 3.66$ & 1.548 & 0.083 & $8.77 \pm 2.23$ & $13.37 \pm 3.15$ & 10.13 & $<0.01$ \\
\hline $\mathrm{Vm}$ & $8.94 \pm 1.87$ & $26.83 \pm 3.36$ & 1.741 & 0.062 & $9.73 \pm 2.21$ & $15.36 \pm 3.29$ & 9.74 & $<0.01$ \\
\hline RI & $1.17 \pm 0.08$ & $0.34 \pm 0.11$ & 1.275 & 0.121 & $1.35 \pm 0.13$ & $0.68 \pm 0.14$ & 9.37 & $<0.01$ \\
\hline
\end{tabular}

Psv, peak systolic blood flow velocity; Vm, mean blood flow velocity; RI, resistance index.

in patients of group B (Table VI). Statistically significant differences were observed between groups $\mathrm{A}$ and $\mathrm{B}$ regarding hemodynamic parameters $\left(\chi^{2}=4.935,7.124 ; 5.478 ; \mathrm{P}<0.05\right)$.

Different types of ocular ischemic diseases have different curative effects. The present study showed that different types of ocular ischemic diseases had different curative effects. EECP had optimum significant effectiveness in ischemic optic neuropathy. Following treatment with EECP and medication for 2 months, no improvement was observed in the visual acuity of patients with clinical symptoms present for $>6$ months, while there was significant improvement in visual fields and optical hemodynamics.

None of the patients with ocular ischemic diseases treated with EECP and medication in groups A and B exhibited any complications during the therapeutic process.

\section{Discussion}

EECP is a non-invasive method used to assist circulation, which improves diastolic augmentation and systolic unloading via a pressurized air cuff around the legs of the patient; cuff pressure was maintained at $\sim 300 \mathrm{mmHg}$ during diastole. In the United States, this method has proven to be effective in chronic angina, as confirmed by large-scale clinical trials (8-11). Findings of those studies suggested that the increase in coronary blood by EECP treatment occurs mainly through diastolic augmentation, which is comparable to the effect on the arterial system by intra-aortic balloon pumping (IABP), although EECP also affects venous return. EECP is beneficial as it can be immediately implemented and is non-invasive without risk of bleeding or infection in contast to IABP. Thus, EECP may be useful to improve circulation in patients with acute heart failure and acute coronary syndrome. In patients with ocular ischemic diseases, EECP induced diastolic augmentation comparable to that of IABP. By contrast, EECP increased preload and the cardiac index $(\mathrm{CI})$ with increased venous return in contrast to IABP (11). In the present study, EECP was performed in patients with ocular ischemic diseases, and the changes in visual acuity, visual fields, as well as the hemodynamic effects were observed in order to investigate the mechanism of EECP and its effects on ocular ischemic diseases (12-14).

Before 1990, the mechanism of this treatment was supposed to promote collateral circulation by increasing diastolic pressure. In their study, Zheng et al identified and confirmed that the flow shear stress was significantly increased during EECP, regulating a series of reactions of shear stress responsive elements, inducing vascular endothelial cell (VEC) repair mechanism and improving VEC function, leading to inhibition of the development of atherosclerosis. A new generation of EECP device was designed based on the abovementioned advances which aimed to promote the arterial flow shear stress more effectively leading to vascular endothelium protection (15-17).

Carotid artery stenosis is a leading cause of ischemic stroke and patients usually present to neurological clinics with stroke symptoms of hemiplegia and homonymous hemianopia (18). An increasing number of patients with carotid artery stenosis have presented to ophthalmological clinics due to eye discomfort. Therefore, such patients who have carotid artery stenosis accompanied by ocular ischemic presentations have received increasing attention (Fig. 1) $(16,19,25)$.

EECP has been used in ischemic disease such as retinal arterial obstruction, ischemic optic neuropathy and optic 


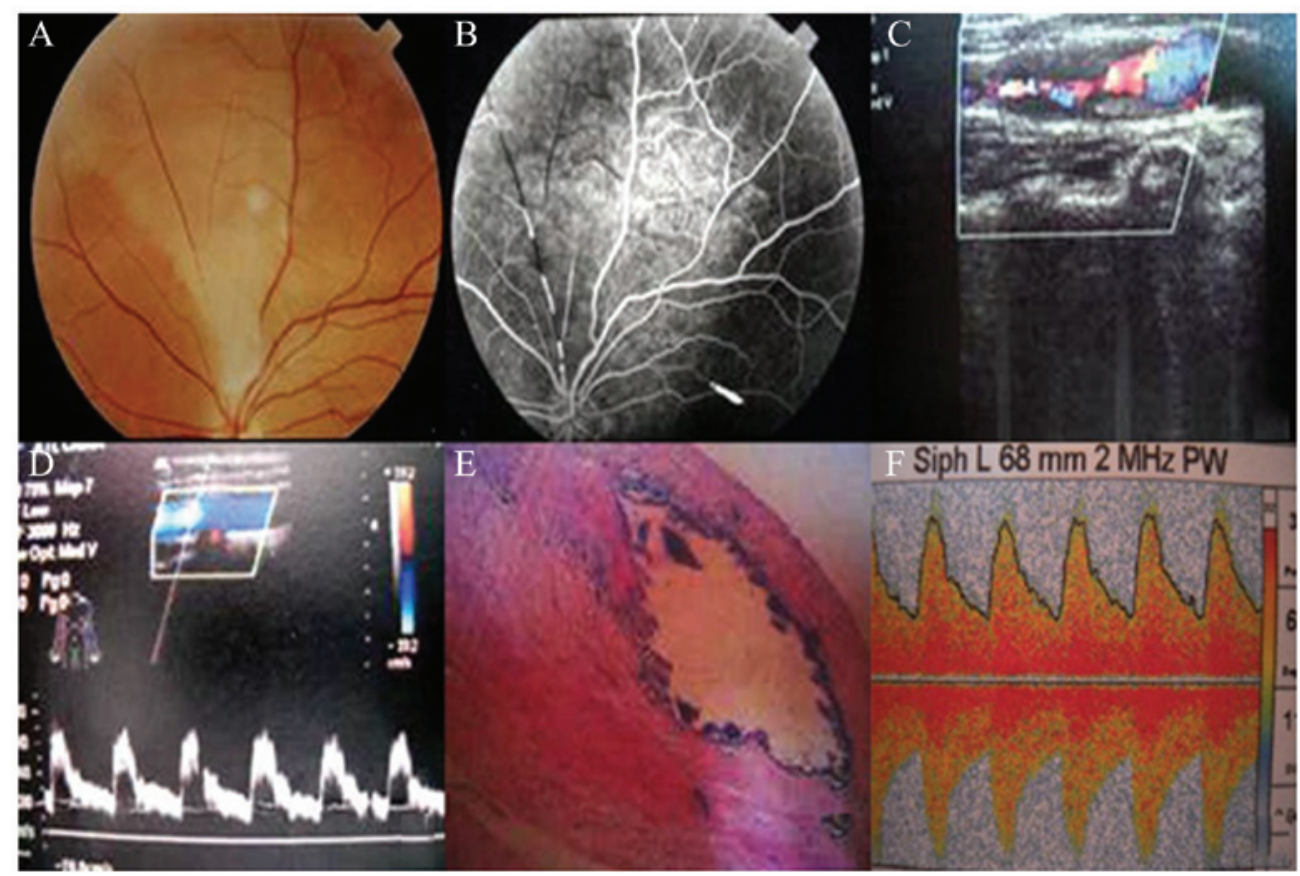

Figure 1. Parts (A-E) were taken from Luo et al (24). Retinal branch artery occlusion caused by carotid artery stenosis (male, 67-year-old patient). (A) Image of the fundus showing occlusion in the retinal branch artery. (B) FFA showing significantly prolonged fluorescein filling time in upper retinal branch artery. (C) Color Doppler ultrasound showing blood flow disturbance in carotid artery secondary to carotid artery stenosis. (D) Carotid artery color Doppler ultrasound showing patent carotid artery lumen after EECP. (E) Pathology of carotid endarterectomy shows atherosclerosis and the resulting intima calcification of the internal carotid artery (H\&E staining; magnification, $\mathrm{x} 40$ ). (F) Transcranial Doppler (TCD) showing blood flow disturbance in carotid artery secondary to carotid artery stenosis.

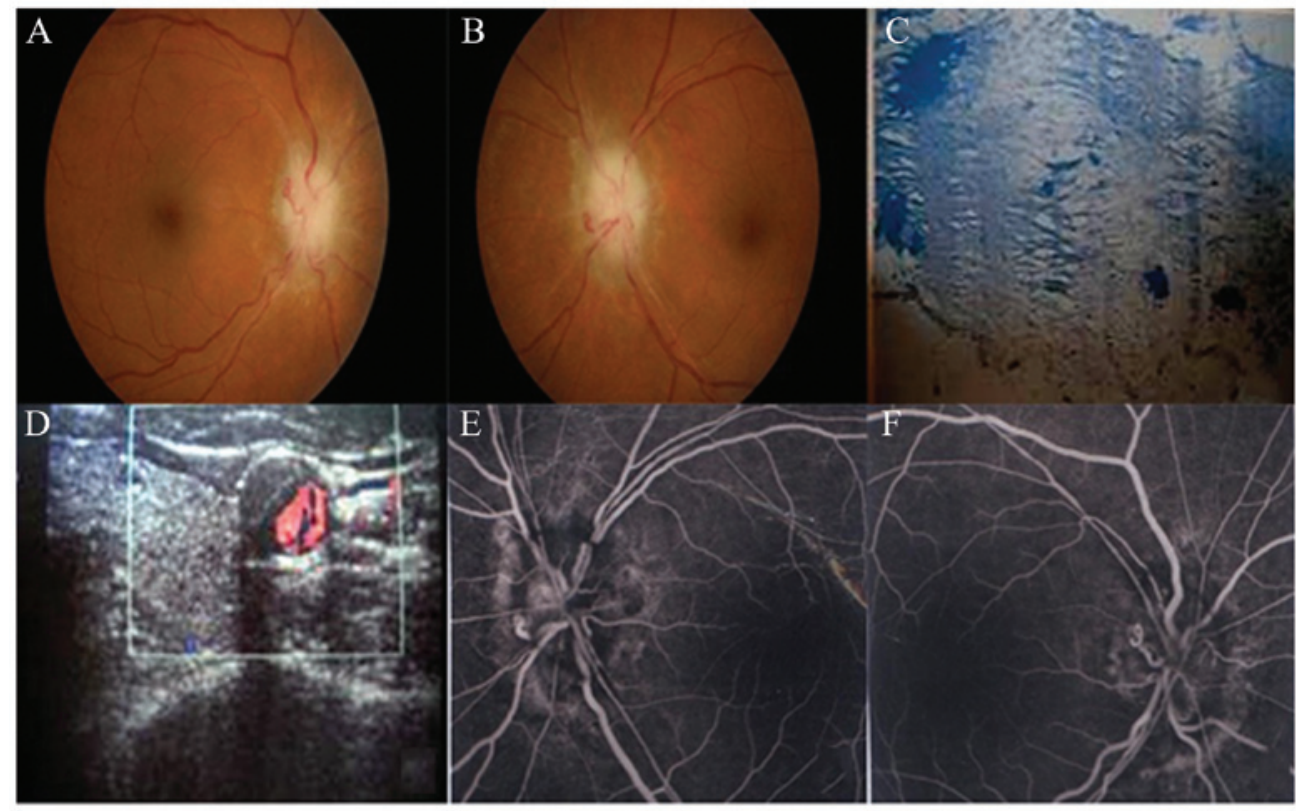

Figure 2. Part (C) was taken from Luo et al (24). Ischemic optic nerve disorders caused by carotid artery stenosis (female, 63-year-old patient). (A and B) Image of the fundus showing optic disc pallor in the ischemic optic nerve disorders. (C) Pathology of carotid endarterectomy shows fibrotic thickening and hyaline changes in arterial wall, and slit-like cholesterol crystal (H\&E staining; magnification, x100). (D) Color Doppler ultrasound shows arterial thrombosis in carotid artery secondary to carotid artery stenosis. (E and F) FFA showing fuzzy optic disc border, tapering optic disc artery, venous tortuosity and dilatation in ischemic optic nerve disorders.

atrophy in the Department of Ophthalmology, the First Affiliated Hospital of Sun Yat-Sen University, and has been proven to have a significant curative effect. Optic nerves are the nerve endings of cerebrum. During the process of optic atrophy, some optic nerve fibers are reversible, and their function improves with prompt recovery of blood supply. EECP can oppress limbs during the relaxation period, increase the blood flow of carotid and vertebral arteries, as well as increase the volume of blood flow of the ophthalmic artery. EECP simultaneously increases the perfusion pressure of 
posterior ciliary arteries and blood supply of the optic nerve, decreases ischemia and the poor supply of oxygen in the optic nerve. As a result, EECP gradually improves the visual function, and significantly improves the visual acuity and field of vision of patients (Fig. 2) (17,20,25).

In their study, Costa et al (21) reported that EECP can promote ramus anastomoticus of retinal ischemia ischemic zone open and collateral circulation formation, improve retinal blood stream, ultimately leading to the treatment of optic atrophy. The present study has shown that there was significant improvement in patients with optic atrophy treated with EECP combined with drugs.

The present study also identified a close positive correlation between curative effect and course of disease in the patients of group A $(\mathrm{R}=0.719, \mathrm{P}<0.05)$. Correlation analysis revealed that the patients with a shorter course of disease exhibited an improved curative effect. Consequently, early diagnosis and intervention are needed in patients with ocular ischemic diseases. Significant improvement in visual acuity and visual fields was observed in some patients with optic atrophy with symptoms present for $>1$ year, following treatment with EECP and medication for several months. The color of video disc also changed from pallor to reddish when under examination using an ophthalmoscope. Therefore, even low-vision patients with long-term optic atrophy may benefit from EECP.

Results of the present study have shown that there was significant improvement in the visual acuity, visual fields and optical hemodynamics of the patients in group A, where statistically significant differences were observed between groups A and B. Moreover, this study demonstrated that patients with ocular ischemic diseases can be treated with EECP 1 or 2 times/day, for $1 \mathrm{~h}$ each time, initially at a course of treatment of half a month, and subsequently for $>1$ month. Younger patients exhibited an improved curative effect compared to older patients, since they had higher diastolic blood pressure when treated with EECP. Therefore, younger patients with ocular ischemic diseases can be treated with EECP 2 times/day using an ophthalmoscope.

The present study has shown that EECP is safe and effective, and that it can be used to treat various conditions apart from hemorrhagic disease, severe hypertension, aortic incompetence, thrombophlebitis and glaucoma. Early intervention may therefore be effective in the treatment of carotid artery stenosis and reduce the incidence of ophthalmic and cerebral complications when treated with EECP combined with drugs (22-24).

\section{References}

1. Zheng ZS: The past, present and future of external counterpulsation. J Sun Yat-Sen Univ Med Sci 27: 602-604, 2006 (In Chinese).

2. Lu L, Wu WK, Zheng ZS, et al: Effects of external counterpulsation on renin-angiotensin system and hemodynamics in dogs with myocardial ischemia. Chin J Pathophysiol 17: 804-807, 2002 (In Chinese).

3. Chen XL, Liu TT, Yu ZH, et al: Enhanced external counterpulsation improved no-reflow phenomena in myocardium after percutaneous coronary intervention. Chin J Hypertens 16: 712-715, 2008 (In Chinese).
4. Lawrence PF and Oderich GS: Ophthalmologic findings as predictors of carotid artery disease. Vasc Endovascular Surg 36: 415-424, 2002.

5. Takaki Y, Nagata M, Shinoda K, et al: Severe acute ocular ischemia associated with spontaneous internal carotid artery dissection. Int Ophthalmol 28: 447-449, 2008.

6. Wang YL, Zhao L, Huang YX, et al: Clinical characteristics of ocular ischemia syndrome. Zhonghua Yan Ke Za Zhi 45: 1080-1083, 2009 (In Chinese).

7. Liu SR, Luo RJ, Li XM, et al: Analysis of ocular ischemic diseases caused by carotid artery stenosis. Chin J Ocul Fund Dis 26: 310-313, 2010 (In Chinese).

8. Li MY and Li CM: Effectiveness of external counterpulsation in the treatment of optic atrophy. Chin J Rehabil Med 18: 101-102, 2003.

9. Wu GF, Du ZM, Hu C, et al: Angiogenic effects of long-term enhanced external counterpulsation in a dog model of myocardial infarction. Am J Physiol Heart Circ Physiol 290: H248-H254, 2006.

10. Wu GF, Du ZM, Hu CH, et al: Microvessel angiogenesis induced by enhanced external counterpulsation in chronic experiment infarct model. J Card Fail 8: S26, 2002.

11. Taguchi I, Ogawa K, Kanaya T, Matsuda R, Kuga H and Nakatsugawa M: Effects of enhanced external counterpulsation on hemodynamics and its mechanism. Circ J 68: 1030-1034, 2004.

12. Shea ML, Conti CR and Arora RR: An update on enhanced external counterpulsation. Clin Cardiol 28: 115-118, 2005.

13. Loh PH, Louis AA, Windram J, et al: The immediate and long-term outcome of enhanced external counterpulsation in treatment of chronic stable refractory angina. J Intern Med 259: 276-284, 2006

14. Lawson WE, Hui JCK, Kennard ED, et al: Two-year outcomes in patients with mild refractory angina treated with enhanced external counterpulsation. Clin Cardiol 29: 69-73, 2006.

15. Amselem L, Montero J, Diaz-Llopis M, Pulido JS, Bakri SJ, Palomares P and Garcia-Delpech S: Intravitreal bevacizumab (Avastin) injection in ocular ischemic syndrome. Am J Ophthalmol 144: 122-124, 2007.

16. Malhotra R and Gregory-Evans K: Management of ocular ischemic syndrome. Br J Ophthahnol 184: 1428-1431, 2000.

17. Karacostas D, Terzidou C, Voutas S, Rafou J, Artemis N and Georgiadis N: Isolated ocular ischemic syndrome with no cerebral involvement in common carotid artery occlusion. Eur J Ophthalmol 11: 97-101,2001.

18. Chen CS and Miller NR: Ocular ischemic syndrome: review of clinical presentation, etiology, investigation, and management. Compr Ophthalmol Update 8: 17-28, 2007.

19. Albers GW, Hart RG, Lutsep HL, Newell DW and Sacco RL: Addendum to the supplement to the guidelines for the management of transient ischemic attacks. Stroke 31: 1001, 2000.

20. Wolintz RJ: Carotid endarterectomy for ophthalmic manifestations: is it ever indicated? J Neuroophthalmol 25: 299-302, 2005.

21. Costa VP, Kuzniec S, Molnar LJ, Cerri GG, Puech-Leão P and Carvalho CA: The effects of carotid endarterectomy on the retrobulbar circulation of patients with severe occlusive carotid artery disease. An investigation by color Doppler imaging. Ophthalmology 106: 306-310, 1999.

22. Sivalingam A, Brown GC and Magargal LE: The ocular ischemic syndrome. III. Visual prognosis and the effect of treatment. Int Ophthalmol 15: 15-20, 1991.

23. Pecold-Stepniewska H, Karolczak-Kulesza M, Wasilewicz R, Krasiński Z and Kulesza J: Glaucoma and ocular ischemic syndrome - case report. Klin Oczna 106: 258-260, 2004 (In Polish).

24. Klijn CJ, Kappelle LJ, van Schooneveld MJ, Hoppenreijs VP, Algra A, Tulleken CA and van Gijn J: Venous stasis retinopathy in symptomatic carotid artery occlusion: prevalence, cause, and outcome. Stroke 33: 695-701, 2002.

25. Luo R, Liu, S, Li X, Zhuo Y and Tian Z: Fifty-eight cases of ocular ischemic diseases caused by carotid artery stenosis. Chin Med J 123: 2662-2665, 2010. 